OPEN ACCESS

Edited by:

Jang Won Yoon,

Kangwon National University,

South Korea

Reviewed by:

Wen-Ching Wang,

National Tsing Hua University, Taiwan

Matthew Cabeen,

Oklahoma State University,

United States

${ }^{*}$ Correspondence:

Dongjin Park

djpark@unl.edu

Kwangcheol Casey Jeong kcjeong@ufl.edu

Specialty section:

This article was submitted to Molecular Bacterial Pathogenesis,

a section of the journal

Frontiers in Cellular and Infection

Microbiology

Received: 13 December 2019

Accepted: 07 May 2020

Published: 05 June 2020

Citation:

Teng L, Lee S, Park $D$ and Jeong KC

(2020) Genetic and Functional

Analyses of Virulence Potential of an

Escherichia coli 0157:H7 Strain

Isolated From Super-Shedder Cattle.

Front. Cell. Infect. Microbiol. 10:271.

doi: 10.3389/fcimb.2020.00271

\section{Genetic and Functional Analyses of Virulence Potential of an Escherichia coli 0157:H7 Strain Isolated From Super-Shedder Cattle}

\author{
Lin Teng ${ }^{1,2}$, Shinyoung Lee ${ }^{1,2}$, Dongjin Park ${ }^{3 *}$ and Kwangcheol Casey Jeong ${ }^{1,2 *}$ \\ ${ }^{1}$ Emerging Pathogens Institute, University of Florida, Gainesville, FL, United States, ${ }^{2}$ Department of Animal Sciences, \\ University of Florida, Gainesville, FL, United States, ${ }^{3}$ Food Science and Technology Department, University of \\ Nebraska-Lincoln, Lincoln, NE, United States
}

Shiga toxin (Stx)-producing Escherichia coli (STEC) O157:H7 is an enteric pathogen that causes life-threatening disease in humans, with cattle being major natural reservoirs. A group of STEC 0157:H7 with a dramatic combination of high virulence potentials and super-shedder bovine origin have been isolated. Here, an STEC O157:H7 isolate, JEONG-1266, was analyzed by comparative genomics, stx genotyping, and phenotypic analyses. The phylogenetic typing and whole-genome comparison consistently showed that JEONG-1266 is genetically close to EC4115 (one of 2006 Spinach outbreak isolates) and SS17 (an isolate from super-shedder cattle) strains, all of which belong to lineage $\mathrm{I} / \mathrm{II}$ and Clade 8 . Both lineage $\mathrm{I} / \mathrm{II}$ and Clade 8 are known to be mostly associated with clinical strains with high virulence and severe clinical symptoms. Further, JEONG-1266, like EC4115 and SS17, harbors stx2a/stx2c genes, and carries Stx-encoding prophages, specifically the $\varphi s t x 2 a-\gamma$ subtype. Possession of the $\varphi s t x 2 a-\gamma$ subtype of Stx-encoding prophages and production of Stx2a have been shown to be a key signature associated with hypervirulent STEC 0157:H7 strains. In silico virulence typing elucidated JEONG1266, EC4115, and SS17 shared a highly conserved profile of key virulence genes at the nucleotide sequence level. Consistently, phenotypic data showed that JEONG-1266 expressed a high level of Stx2 toxins and had the full capacity of adhesion in vitro. Taken together, our study suggests that JEONG-1266 may represent an emerging STEC O157:H7 group, which are hypervirulent strains that originate from super-shedders, that can be a threat to food safety and public health.

Keywords: virulence, comparative genomics, super-shedder, functional analysis, E. coli 0157:H7

\section{INTRODUCTION}

Shiga toxin (Stx)-producing Escherichia coli (STEC) O157 has become a major public health burden worldwide (Arthur et al., 2013; Munns et al., 2015), especially in Japan, Scotland and North America (Mead et al., 1999; Sakuma et al., 2006; Pollock et al., 2007), since it was first identified as a foodborne pathogen in 1982. E. coli O157 infection in humans mainly occurs through consuming food products contaminated with this pathogen (Griffin and Tauxe, 1991; Caprioli et al., 2005; CDC, 2006) and can develop a range of symptoms such as bloody 
diarrhea and life-threatening hemolytic-uremic syndrome (HUS) (Nataro and Kaper, 1998; Paton and Paton, 1998; Gyles, 2007). During the decade from 2003 to 2012, a total number of 390 E. coli O157 outbreaks, including 353 O157 outbreaks, were identified and reported in the United States (Heiman et al., 2015). E. coli $\mathrm{O} 157$ infections resulted in an average of 63,153 acute cases annually, leading to $\sim 254$ million US dollars for the annual cost of illness treatments (Hoffmann et al., 2012).

Cattle are the principal asymptomatic reservoirs of this pathogen (Wells et al., 1991). However, the excretion rate of $E$. coli $\mathrm{O} 157$ drastically varies among individual cattle, ranging from $10^{2}$ to more than $10^{7} \mathrm{CFU} / \mathrm{g}$ of feces (Chase-Topping et al., 2007; Jeon et al., 2013). Among these cattle, a subset of them shedding E. coli $\mathrm{O} 157$ at levels $>10^{4} \mathrm{CFU} / \mathrm{g}$ in feces are defined as "supershedders" (Matthews et al., 2006). It has been reported that $20 \%$ of most infectious cattle were responsible for about $80 \%$ of $E$. coli O157 transmission between animals in Scotch farms (Matthews et al., 2006). Jeon et al. reported super-shedders disseminated $E$. coli $\mathrm{O} 157$ to other cattle housed in the same pen, causing the E. coli $\mathrm{O} 157$ shed by super-shedders predominant in the farm (Jeon et al., 2013). Ecological dynamics of these predominant E. coli $\mathrm{O} 157$ and super-shedders increase the frequencies of contamination in animal food products such as beef and milk (Gyles, 2007). Hence, it is of great importance to understand the characteristics of the E. coli $\mathrm{O} 157$ strains from the super-shedders to reduce the prevalence of this pathogen in food-producing animals and consequently to curtail the number of foodborne outbreaks and human illnesses.

The pathogenicity of STEC O157 is determined by its key virulence factors, including Stxs, adhesins, and the type III secretion system (T3SS) and its effectors. Stx, a member of the AB-type toxin family, is the major virulence factor of STEC O157 and uses globotriaosylceramide (Gb3), which is rich in human endothelial cells, as their receptor to gain intracellular translocation (Melton-Celsa, 2014). Stx-medicated cytotoxicity is mainly based on inhibition of protein synthesis on the host target cells by depurinating a specific adenine residue of the $28 \mathrm{~S}$ rRNA (Croxen et al., 2013). Stxs consist of two major types, Stx1 and Stx2, each of which has variant subtypes. Stx1, Stx2a, and Stx $2 c$, alone or in combination, are the most clinically relevant subtypes. In particular, Stx $2 \mathrm{a}$ has been shown to be associated with more severe clinical symptoms than Stx1 or Stx2c (Boerlin et al., 1999). The stx genes are invariably encoded by a lambdoid prophage in STEC O157 and their production is tightly coupled with phage induction (Tyler et al., 2004). In particular, the level of Stx2 production is a critical virulence parameter for STEC O157 but is known to vary remarkably between STEC O157 strains. A recent study showed that variation in the Stx 2 production level in STEC O157 strains is correlated with particular subtypes of Stx-encoding prophages (Ogura et al., 2015).

Intimin and Tir (translocated intimin receptor), encoded by eae and tir genes, respectively, are a critical adhesin/receptor pair in STEC O157, as they enable the pathogen to intimately bind the intestinal epithelium of the host, resulting in the characteristic attachment and effacement lesion (Melton-Celsa et al., 2012). Several studies with animal models have shown that the intimin/Tir function is essential for stable colonization of STEC O157 in host cells (Mckee et al., 1995; Cornick et al., 2002; Sheng et al., 2006). STEC O157 uses the T3SS, a needle-like structure, to directly translocate Tir and other T3SS effectors inside of the intestinal epithelium of the host. These T3SS effectors, subsequently, manipulate signaling pathways in host cells and establish infection (Melton-Celsa et al., 2012).

STEC O157 have been classified based on several methods including Clade typing (Manning et al., 2008), Lineages classification (Yang et al., 2004), IS-based classification (Stanton et al., 2014), and phylogenetic analysis (Shridhar et al., 2018). Three primary evolutionary lineages of STEC O157 include lineage I (typically from human clinical and bovine sources), lineage II (predominantly from bovine source), and lineage I/II (related to human infection and outbreaks) (Kulasekara et al., 2009; Laing et al., 2009; Stanton et al., 2014). Lineage I/II and Clade 8, in particular, have been shown to be mostly associated with clinical strains with high virulence and severe clinical symptoms. The Stx genotype is one of the conserved characteristics that are associated with those classification schemes; especially, Clade 8 has been proposed to be a highly pathogenic clade of STEC O157 and typically carries stx2c/stx2a (Manning et al., 2008; Iyoda et al., 2014).

Besides in silico genotyping and comparative genomics, phenotypic and functional characterizations of various key virulence factors are required to fully investigate the virulence potential of STEC O157. Park et al. reported that inactivation of stx2 gene by the insertion of an IS mobile element can be found naturally in E. coli O157 isolates, turning the Shiga toxigenic E. coli O157 strain into a non-Shiga toxigenic strain (Park et al., 2013). The same report also showed that an intact stx gene encoded in uninducible Stx-prophages was not fully expressed. Therefore, western blotting is necessary to determine the production of intact Stxs in E. coli O157 strains. The adhesion of STEC O157 to the human intestinal epithelium is a crucial step for its pathogenicity (Grys et al., 2005).

Despite that STEC O157 from super-shedder cattle have been isolated and investigated in several studies, the complete whole-genome sequences of these isolates are limited in NCBI database and their virulence potential remains unclear. In our previous study, an E. coli O157 strain, JEONG-1266, from super-shedder cattle was sequenced using PacBio sequencing method (Teng et al., 2016). In the current study, we performed a comprehensive phylogenetic analysis, comparative genomics, and functional analyses to investigate the virulence potential of JEONG-1266. Our data support that JEONG-1266 may represent a hypervirulent STEC O157 that is associated with supershedder cattle.

\section{MATERIALS AND METHODS}

\section{Bacterial Strains}

The E. coli O157:H7 strain JEONG-1266 was isolated from the recto-anal junction (RAJ) of a super-shedder cattle located in Florida (Jeon et al., 2013). Genomes of other three E. coli O157:H7 strains, EDL933, EC4115, and SS17, were used as reference genomes for comparative analyses. EDL933 
(ATCC43895) [NC_CP008957] was linked to a hamburgerborne outbreak in 1982 in the U.S. The clinical isolate, EC4115 [NC_011353], was isolated from a human at the time of 2006 spinach outbreak in Maine, U.S (Eppinger et al., 2011). Another reference strain, SS17 [NZ_CP008805], was collected from the RAJ of super-shedder cattle (Cote et al., 2015).

\section{Genomic DNA Extraction}

Genomic DNA of JEONG-1266 was isolated using QIAGEN DNA mini Kit. Briefly, $1 \mathrm{~mL}$ overnight culture of JEONG1266 was centrifuged at $5,000 \times g$ for $10 \mathrm{~min}$. Following the manufacturer's protocol, the bacterial pellet was resuspended in buffer ATL and treated with $20 \mu \mathrm{L}$ of proteinase $\mathrm{K}$ at $56^{\circ} \mathrm{C}$ for $30 \mathrm{~min}$. The released DNAs were eluted with $100 \mu \mathrm{L}$ of $\mathrm{ddH}_{2} \mathrm{O}$.

\section{Stx2 Subtyping}

Polymerase chain reaction (PCR) was performed to determine the stx2 subtypes (i.e., st $2 a$ and st $2 c$ gene) carried by JEONG-1266. Two E. coli O157:H7 strains, EDL933 carrying st $2 a$ and stx1 and FRIK2455 carrying st $2 c$, were used as controls. Primers used for detecting st $2 a$ (stx2a-F: $5^{\prime}$-CTTTTCGACCCAACAAAGTTATGT-3' and stx2a-R: $5^{\prime}$-CACAGTCCCCAGTATCGCT- $\left.3^{\prime}\right)$ and st $2 c$ (stx2c-F: $5^{\prime}$-TACTGTGCCTGTTACTGGGC-3' and stx2c-R: $5^{\prime}$ ACAGTGCCCAGTATCGCC-3') were designed according to previous study (Jeon et al., 2013). The PCR reaction condition was set as $94^{\circ} \mathrm{C}$ for $5 \mathrm{~min} ; 94^{\circ} \mathrm{C}$ for $30 \mathrm{sec}, 54^{\circ} \mathrm{C}$ for $30 \mathrm{sec}, 72^{\circ} \mathrm{C}$ for $60 \mathrm{~s}$ for 30 cycles and a final extension time of $10 \mathrm{~min}$ at $72^{\circ} \mathrm{C}$. Amplified PCR product was analyzed in $1 \%$ agarose gel electrophoresis and visualized by ethidium bromide staining.

\section{Phage Induction}

The method of phage induction was previously described (Park et al., 2013). Briefly, cell lysis caused by phage induction was measured by optical density (OD). Overnight culture of bacterial strains was inoculated into Luria-Bertani (LB) broth. Mitomycin $\mathrm{C}(\mathrm{MMC})$ treatment $(0.5 \mu \mathrm{g} / \mathrm{mL})$ was followed when the $\mathrm{OD}_{600}$ of the cell culture reached 0.7. EDL933 and DH5 $\alpha$ were used as a positive and a negative control, respectively, for phage induction. For phage particle preparation, MMC was added to $25 \mathrm{~mL}$ of cell culture when $\mathrm{OD}_{600}$ reached 0.7 to make a final concentration of $1 \mu \mathrm{g} / \mathrm{mL}$. After $18 \mathrm{~h}$ incubation, unlysed cells and debris were removed by centrifugation at 3,700 $\times g$ for $20 \mathrm{~min}$ at $4^{\circ} \mathrm{C}$, and the resultant supernatant was filtered with $0.22 \mu \mathrm{m}$-pore-size membrane (Fisher Scientific, USA). Precipitation of phage particles was performed using 0.25 volume of polyethylene glycol/ $\mathrm{NaCl}$ solution (20\% polyethylene glycol 8,000 and $10 \% \mathrm{NaCl}$ ). The polyethylene glycol/ $\mathrm{NaCl}$ solution containing phage particles was incubated at $4{ }^{\circ} \mathrm{C}$ overnight, followed by centrifugation at $12,000 \times g$ for $1 \mathrm{~h}$. The pellet was resuspended and stored in $1 \mathrm{~mL}$ SM buffer $(0.58 \mathrm{~g} \mathrm{NaCl}, 0.2 \mathrm{~g}$ $\mathrm{MgSO}_{4} \cdot 7 \mathrm{H}_{2} \mathrm{O}, 0.01 \mathrm{~g}$ Gelatin per $100 \mathrm{~mL}$ of $1 \mathrm{M}$ Tris-Cl pH7.5). SDS-PAGE was used to check the phage protein profile after an aliquot of the phage suspension was mixed with SDS-loading buffer and boiled for $5 \mathrm{~min}$.

\section{Western Blot}

To detect the expression of Stx2, Western blot was conducted as previously described (Jeon et al., 2014). Briefly, the exponential phase culture $\left(\mathrm{OD}_{600}=0.7\right)$ of an E. coli $\mathrm{O} 157$ strain was treated with $\mathrm{MMC}$ to the final concentration of $1 \mu \mathrm{g} / \mathrm{mL}$ to induce the lytic cycle of phages in the E. coli O157 strain and concomitant stx gene expression. Next, the $\mathrm{O} 157$ cell culture was incubated for $18 \mathrm{~h}$ for complete cell lysis. The cell debris was removed by centrifugation $\left(3,700 \times g\right.$ for $20 \mathrm{~min}$ at $\left.4^{\circ} \mathrm{C}\right)$ and the cell-free supernatant was collected by using $0.22 \mu \mathrm{m}$-poresize membrane filter (Fisher Scientific, USA). The total proteins in the supernatant were precipitated by adding 0.25 volume of $100 \%$ trichloroacetic acid (Fisher Scientific, USA) to the cellfree culture. The mixture was incubated on ice for $30 \mathrm{~min}$ and centrifuged at $12,000 \times \mathrm{g}, 4^{\circ} \mathrm{C}$ for $30 \mathrm{~min}$. The protein pellet was finally washed using cold acetone. The total proteins were separated by $12 \%$ sodium dodecyl sulfate-polyacrylamide gel and transferred to an Immobilon-P polyvinylidene difluoride membrane with a pore size of $0.45 \mu \mathrm{m}$ (MilliporeSigma, USA) for Western blot. The membrane was blocked using 5\% skim milk (BD, USA) in TBST (10 mM Tris-HCL [pH 7.4], $150 \mathrm{mM}$ $\mathrm{NaCl}$, and $0.05 \%$ Tween-20) at room temperature for $1 \mathrm{~h}$. Later, the membrane was incubated overnight at $4^{\circ} \mathrm{C}$ with monoclonal Verotoxin II-a subunit antibody (Meridian Life Science Inc., USA), and washed using TBST at room temperature for $1 \mathrm{~h}$. The membrane was then incubated with HRP conjugated secondary antibody (GE Healthcare, USA) diluted 1:10,000 in TBST, washed with TBST, and incubated with a chemiluminescent substrate (GE Healthcare, USA). Finally, the membrane was exposed to Kodak BioMax film (Sigma, USA).

\section{Adherence Assay to HEp-2 Cells}

The ability of JEONG-1266 to adhere to human epithelial type2 (HEp-2) cells was evaluated. EDL933 was used as a positive control and DH5 $\alpha$ was used as a negative control. HEp-2 cells were maintained in Dulbecco's Modified Eagle Medium (DMEM; Coring, USA) composed of $10 \%$ (vol/vol) heat-inactivated fetal bovine serum at $37^{\circ} \mathrm{C}$ and $5 \% \mathrm{CO}_{2}$. Approximately $1 \times 10^{5}$ HEp-2 cells were seeded into a 24 -well polystyrene plate as measured using a hemocytometer and allowed to grow until $90 \%$ confluence. Bacterial cultures were grown overnight at $37^{\circ} \mathrm{C}$ in LB broth and washed with sterile phosphate-buffered saline (PBS) three times. The final cell pellet was resuspended in PBS to a final concentration of $\mathrm{OD}_{600}=1\left(5 \times 10^{8} \mathrm{CFU} / \mathrm{mL}\right)$. An aliquot $(20$ $\mu \mathrm{L}$ ) of the resuspended culture was added into $480 \mu \mathrm{L}$ of DMEM. Then $500 \mu \mathrm{L}$ of DMEM containing $1 \times 10^{7}$ CFU of bacteria was added to each well to obtain a multiplicity of infection (MOI) of 100 . After a 3 -h incubation at $37^{\circ} \mathrm{C}$ with $5 \% \mathrm{CO}_{2}$, the bacterial suspension was removed and $500 \mu \mathrm{L}$ of new DMEM was added to each well for another 3-h incubation. The infected cell monolayer was washed using sterile PBS three times to remove any unattached bacteria. To detach the HEp-2 cells from the plate, $1 \mathrm{~mL}$ of $0.1 \%$ Triton X-100 in PBS buffer was added to each well and allowed to incubate for $5 \mathrm{~min}$. The media was collected from each well, serially diluted in LB broth, and plated on LB agar plates to enumerate the number of attached bacterial cells. The adherence assay of each strain was conducted in triplicate. 
Statistical significance between the level of attachments of isolates was accessed using a student's $t$-test $(\alpha=0.05)$.

\section{Comparative Genomics}

The complete genome of JEONG-1266 was acquired using PacBio sequencing and annotated by NCBI annotation pipeline (Teng et al., 2016). The completed genome was circularly presented by CGview (Stothard and Wishart, 2005; Overbeek et al., 2014; Brettin et al., 2015). All the coding sequences (CDSs) were assigned to different subsystems based on their functions using RAST (Overbeek et al., 2014; Brettin et al., 2015). Mauve 2.3.1 with default setting (Darling et al., 2004, 2010) was employed to compare the whole-genome architecture of JEONG1266 and reference strains. Pangenome analysis was conducted to identify unique genes among strains using Prokka annotation and Roary (Seemann, 2014; Page et al., 2015). To identify the position of prophage related genes, the PHAge Search Tool (PHAST) (http://phast.wishartlab.com/) (Zhou et al., 2011) with default setting was used. The positions of predicted prophage regions of JEONG-1266, as well as reference genomes, were presented using BLAST Ring Image Generator (BRIG) (Alikhan et al., 2011). To investigate the similarity of Stx2a-encoding prophages in JEONG-1266 and other three reference strains, the nucleotide sequences of the prophages were compared using BLASTn embedded in EasyFig (Sullivan et al., 2011). Genes encoding known and putative virulence factors in the genome of JEONG-1266 were identified using the Virulence Factor Database (VFDB) (Chen et al., 2016) in Pathosystems Resource Integration Center (PATRIC) (https://www.patricbrc.org/portal/ portal/patric/Home) (Wattam et al., 2014). The false-negative virulence genes were manually curated by doing BLASTn against whole-genome sequences of each isolate. The multilocus sequence typing (MLST) of E. coli can be identified using two different sets of housekeeping genes (Wirth et al., 2006; Jaureguy et al., 2008). Both typing methods were employed by MLST 2.0 in the Center for Genomic of Epidemiology (http://www. genomicepidemiology.org/).

\section{In silico Genotyping}

In silico genotyping was used to predict the pathogenicity of JEONG-1266. Two methods were used to identify the clade of JEONG-1266. An in silico Clade 8 rhs A was used to determine whether the isolate belongs to the Clade 8 based on the presence of a SNP (3468C) in the rhsA gene (Liu et al., 2009). Besides, another in silico analysis based on 32 SNPs was also conducted to determine the clade of JEONG-1266 (Manning et al., 2008). To identify LSPA-6 profile of JEONG-1266, sequences of six pairs of primers were applied for in silico PCR (Yang et al., 2004). An isolate showing an LSPA-6 profile of 111111 was defined as lineage I. A strain with LSPA-6 profile of 222222 or 222223 was defined as lineage II. Other genotypes were defined as lineage I/II (Stanton et al., 2014). The subtype of Stx2-encoding prophage was identified by BLAST the sequence of PCR primers against the nucleotide sequence of the Stx2-encoding prophage (Ogura et al., 2015).

\section{Phylogenetic Analysis}

A phylogenetic tree of 26 E. coli O157: $\mathrm{H} 7$ strains was constructed using Parsnp (Treangen et al., 2014). The numbers of SNPs between strains were calculated using the VCF file generated by Parsnp. Figtree (v.1.4.2) (http://tree.bio.ed.ac.uk/software/ figtree/) was used to edit the phylogenetic tree.

\section{Accession Numbers}

The complete genome sequence of E. coli O157 strain JEONG1266 [NZ_CP014314], EDL933 [NC_CP008957], EC4115 [NC_011353], and SS17 [NZ_CP008805] were deposited in NCBI. The accession numbers of the strains used for comparative genomics were listed in the supplementary data (Table S1).

\section{RESULTS}

\section{A Genomic Overview of JEONG-1266}

The STEC O157:H7 strain JEONG-1266 contained a chromosome of 5,478,683 and a 95,910 bp pO157 plasmid, with a GC content of $50.5 \%$ (Figure 1A, Table 1). Its chromosome was shown to encode for 5,363 CDSs, 106 tRNAs, and 22 rRNAs, while the pO157 contained 100 genes. Compared to the three reference strains of STEC O157:H7 including a super-shedder strain (SS17) and two outbreak-associated strains (EDL933 and EC4115), the chromosome of JEONG-1266 was slightly shorter in size, containing fewer CDSs (Table 1). The number of rRNA genes was highly conserved, while that of tRNA genes differed between the four strains, with the highest tRNA gene number in JEONG-1266. Functional categorization of genes by RAST assigned $57 \%$ (3,082 of 5,363 CDSs) of genes with the 27 subsystems categories, leaving the other $43 \%$ of the genes unclassified (Figure 1B). Among the classified genes, 114 genes belonged to the category of virulence, disease, and defense (Figure 1B), which was focused in this study.

\section{Phylogenetic Characterization of the JEONG-1266 Strain}

SNP-based phylogenetic analysis of JEONG-1266 was performed using the core genome sequences of 26 previously characterized E. coli $\mathrm{O} 157: \mathrm{H} 7$ strains isolated from diverse sources (Table S1). The 26 isolates clustered into three major clades, Clade I, II, and III (Figure 2A). Clade I contained clinical strains that have been associated with outbreaks and severe diseases in humans, while Clade II consisted of strains of bovine origin (FRIK966 and FRIK2000) and ground beef (EC869) (Figure 2A and Table S1). Clade III was composed of both bovine and clinical strains (Figure 2A and Table S1). JEONG-1266 belonged to Clade III, which consisted of 2006 multistate spinach outbreak strains (e.g., EC4115) (Eppinger et al., 2011) and the super-shedder bovine strains (e.g., SS17) (Cote et al., 2015). In particular, the SNP matrix data revealed that JEONG-1266 is most closely related to EC4115 (48 SNPs) and EC4191 (56 SNPs) among the spinach outbreak strains (Figure S1). The MLST profile showed that all of the Clade II and Clade III strains analyzed, including JEONG1266, were typed as ST628 (Figure 2A). Four strains in Clade I (EDL933, Xuzhou21, TW14588, and EC4501) belonged ST822, 

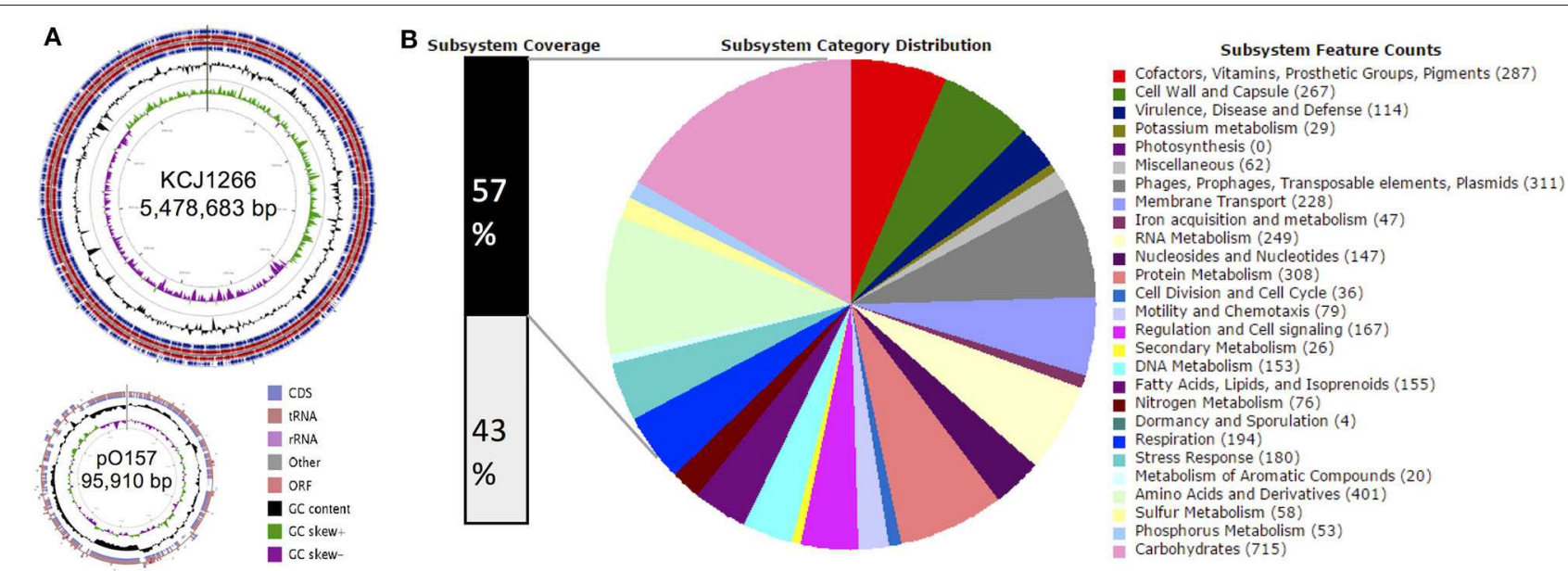

FIGURE 1 | Genomic overview. (A) Genome map of JEONG-1266. The chromosome (top) and plasmid (bottom) of JEONG-1266 were displayed. Marked characteristics are shown from outside (ring 1) to the center (ring 6): Coding Sequences (CDSs), tRNA and rRNA in forward strand (ring1), Open Reading Frames (ORFs) in forward strand (ring 2); ORFs in reward strand (ring 3); CDSs, tRNA and rRNA in reward strand (ring 4), GC content (ring 5), and GC skew (ring 6). (B) Subsystem category distribution. The annotated genes in JEONG-1266 were classified into different subsystem categories based on gene function. The subsystems contain $57 \%$ of total genes (black bar on the left). The other $43 \%$ of genes (white bar on the left) do not belong to any of the subsystems.

TABLE 1 | Genome statistics of JEONG-1266 and reference 0157 strains.

\begin{tabular}{|c|c|c|c|c|}
\hline & $\begin{array}{l}\text { JEONG- } \\
1266\end{array}$ & SS17 & EC4115 & EDL933 \\
\hline $\begin{array}{l}\text { Length of sequence } \\
(\mathrm{Mb})\end{array}$ & 5.48 & 5.52 & 5.57 & 5.53 \\
\hline G+C ratio (\%) & 50.5 & 50.5 & 50.5 & 50.4 \\
\hline $\begin{array}{l}\text { Coding sequence } \\
\text { (CDS) }\end{array}$ & 5,363 & 5,389 & 5,488 & 5,435 \\
\hline No. rRNA & 22 & 22 & 22 & 22 \\
\hline No. tRNA & 106 & 103 & 105 & 101 \\
\hline $\begin{array}{l}\text { Plasmid } \\
\text { (bp; gene number) }\end{array}$ & $\begin{array}{l}\text { p0157 } \\
\text { (95910; 100), }\end{array}$ & $\begin{array}{l}\text { pO157 } \\
(94,645 ; 100), \\
\text { pSS17 } \\
(37,447 ; 43)\end{array}$ & $\begin{array}{l}\text { pO157 } \\
(94644 ; 101), \\
\text { pEC4115 } \\
(37452 ; 43)\end{array}$ & $\begin{array}{l}\text { pO157 } \\
(92076 ; 95)\end{array}$ \\
\hline
\end{tabular}

while the other 2 Clade I strains (Sakai and 1044) displayed a different MLST type, i.e., ST296 (Figure 2A). Therefore, SNPbased phylogeny and MLST together support that JEONG-1266 strain is closely related with STEC O157:H7 from two particular sources; super-shedder bovine and the spinach outbreak.

\section{The Genome Architecture and Prophages}

To further understand the genomic characteristics of JEONG1266, the whole-genome architecture of JEONG-1266 and the other 3 reference strains (SS17, EC4115, and EDL933) were analyzed using Mauve 2.3.1. JEONG-1266 and EC4115 have 7 synteny blocks (SBs), while SS17 and EDL933 have 8 SBs. The EDL933 genome contained inverted SB4 and SB6 (Figure 2B). In addition, an extra non-homology block was found in SS17 and EDL933. Consistent with the phylogenetic analysis results, the genome architecture of JEONG-1266 was most similar to that of EC4115.
E. coli O157:H7 genomes are characterized by the presence of multiple prophages whose sequences are highly variable among strains. Many non-phage genes with virulent functions are present in prophages, including stx and $t c c P$ (an effector for LEE T3SS) (Garmendia et al., 2005). PHAST analysis identified a total of 19 prophages, including 11 intact prophages, in JEONG1266 (Table 2, Figure S2). When the chromosome sequences of JEONG-1266 was compared with that of EC4115 and SS17, it carried 20 and 79 unique genes, respectively (Table S2). These unique genes located in prophage regions, mostly encoding hypothetical proteins or phage-related proteins. Furthermore, genomic variations between JEONG-1266 and the reference strains were mainly detected in prophage regions, P1, P4, P8, P9, and P10 (Table 2, Figure 2B). Except for P10, all the other four variable prophages were predicted to be intact. The genome of JEONG-1266 was shown to have two intact Stx-encoding prophages, P12 and P14, each of which carried stx subtypes, st $x 2 c$ and st $x 2 a$, respectively.

\section{Analysis of Virulence Genes}

Besides stx genes, the presence of other virulence genes was analyzed to address the virulence potential of the JEONG1266 strain. In the four strains, a total of 132 virulence genes were identified using VFDB in PATRIC and categorized into 8 major functional groups: adherence (30 genes), chemotaxis (4 genes), invasion (2 genes), iron binding and uptake (21 genes), toxin (8 genes), protease (2 genes), T3SS (26 genes), and T3SS effectors (40 genes) (Figure 3A). Eight toxin genes were characterized; hly $A B C D$ for hemolysin, $s t x 1 A B$ for Stx1, and st $2 A B$ for Stx2. JEONG-1266, SS17, and EC4115 strains shared a highly conserved virulence profile overall. Most of the virulence genes displayed high homology (>90\%) to the reference genes in the VFDB (Figure 3A). The most divergent gene categories $(<90 \%)$ to the database include adherence and 


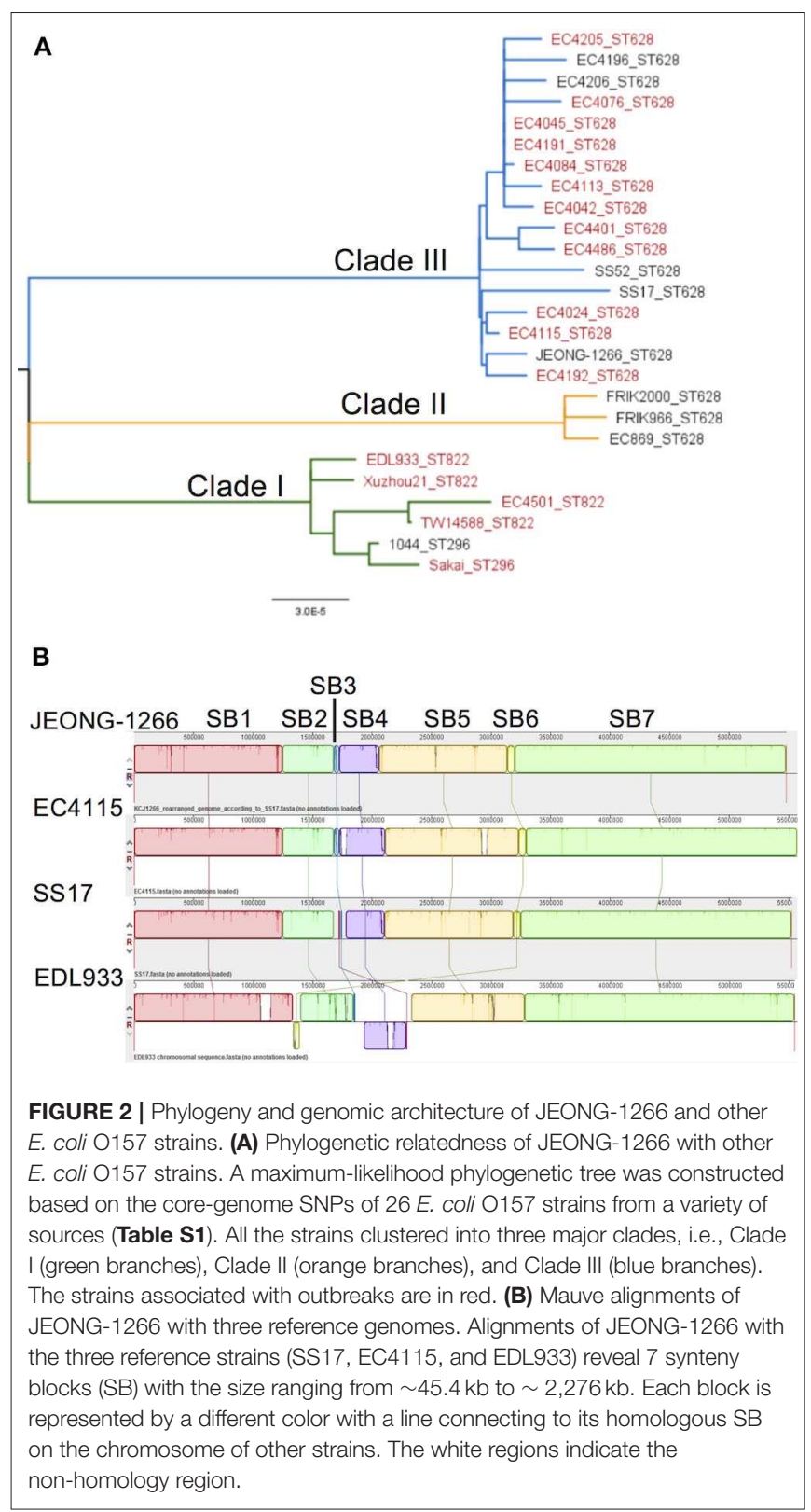

chemotaxis. The adherence-associated genes included intimin (eae), cytotoxin (toxB), outer membrane adhesin Paa (paa), E. coli common pilus (ecpABCDER), curli ( $\operatorname{csg} B E F G$ ), type 1 fimbriae (fimABCDEFGHI), and flagellar (flgCGH and fliGMP). JEONG1266 encoded conserved T3SS structural genes as well as a total of 41 putative T3SS effector genes, indicating the presence of a functional T3SS. The essential genes of the locus of enterocyte effacement (LEE) (Nataro and Kaper, 1998), including tir, eae, $\operatorname{esp} A$, and esp $B$, were shown to be highly conserved. Taken together, these results indicate that JEONG-1266 possesses an array of virulence genes required for the full virulence potential of STEC O157, a characteristic that often lacks in strains of bovine origin.

\section{Determination of Lineage, Clade, and stx Genotype}

Combinational characteristics of the lineage type (I, II, I/II), stx genotype, the insertion site of the Stx-encoding prophage have been shown to be conserved in STEC O157 and useful to determine virulence potential and evolutionary relationship of the pathogen (Stanton et al., 2014). To determine the lineage of JEONG-1266, in silico LSPA-6 typing was performed using nucleotide sequences of the 6 polymorphism markers (Yang et al., 2004). JEONG-1266 showed a lineage I/II profile (i.e., 211111) (Figure 3B), which is identical to those of SS17 and EC4115 (Stanton et al., 2014; Cote et al., 2015).

Clade determination was conducted based on 32 SNP loci on the chromosome of E. coli O157:H7 (Manning et al., 2008). The resultant SNP profile indicated that EDL933 belonged to the Clade 3 while JEONG-1266, EC4115, and SS17 belonged to the Clade 8 (Figure 3B; Table S3). The Clade 8 has been shown to be the most virulent clade of $E$. coli $\mathrm{O} 157: \mathrm{H} 7$ due to high levels of Stx expression and cytotoxicity (Neupane et al., 2011).

Two types of Stx, Stx1, and Stx2, show distinct cytotoxicity and Stx2 is more frequently associated with severe diseases in humans than Stx1 (Melton-Celsa, 2014). In addition, variable Stx2 subtypes have been identified, including Stx2a and Stx2c, and Stx2a is known to be more toxic to humans than Stx2c (Schmitt et al., 1991). Stx2a and Stx2c are typically encoded in different Stx-encoding prophages. In silico analysis showed that both $s t \times 2 a$ and $s t \times 2 c$ are present in JEONG-1266, EC4115, and SS17 but only JEONG-1266 and EC4115 shared the same insertion site for Stx2a-encoding prophage, the $\arg W$ locus (Figure 3B). SS17 and EDL933 used yehW and wrbA loci as the insertion site, respectively. These results suggest that the Stx2a-encoding prophage of JEONG-1266 is more related to that of EC4115.

Taken together, combinational genetic characteristics of JEONG-1266 (i.e., the lineage/clade types, stx genotype, and the insertion site of the Stx-encoding phage) further support its high virulence potential, especially being a member of the hypervirulent Clade 8 with the stx2a/stx2c genotype.

\section{Characterization of Stx2a-encoding Prophage Subtype}

Of the two types of Stx2, Stx2a and Stx2c, Stx2a is known to as a major risk factor for severe STEC infections. Ogura et al. identified four major subtypes of Stx2a-encoding prophage $(\alpha$, $\beta, \gamma$, and $\delta)$ and found that strains carrying $\varphi s t x 2 a-\gamma$ or $\varphi s t x 2 a-$ $\alpha$ (particularly the former) can produce higher Stx2 levels than strains carrying other stx2a phage subtypes (Ogura et al., 2015).

The subtype of Stx2a-encoding prophage of JEONG-1266 was determined by in silico PCR analysis, using the nested primer sets designed for Stx2a-encoding prophage subtyping (Ogura et al., 2015). The results showed that Stx2a-encoding prophage carried by JEONG-1266 is $\varphi s t x 2 a-\gamma$. Since both EC4115 and EDL933 have been shown to carry $\varphi$ stx2a- $\gamma$ (Ogura et al., 2015), the genome structures of the Stx2a-encoding prophage were compared among JEONG-1266, SS17, EC4115, and EDL933. The length of the Stx2a-encoding prophage in JEONG-1266 
TABLE 2 | Genome encoded in the 19 phage regions of JEONG-1266.

\begin{tabular}{|c|c|c|c|c|}
\hline Phage regions & Size (Kbp) & Completeness & Synteny block & Encoded genes \\
\hline P1 & 26.5 & Intact & SB1 & Phage DNA invertase; Phage integrase; and phage assembly protein \\
\hline P2 & 38.5 & Intact & SB1 & Lysozyme and Attachment invasion locus protein precursor \\
\hline P3 & 38 & Incomplete & SB1 & Phage assembly proteins and 3 tRNAs \\
\hline P4 & 29.4 & Intact & SB1 & Attachment invasion locus protein precursor and phage lysis \\
\hline P5 & 50.5 & Intact & SB2 & Integrase and phage assembly proteins \\
\hline P6 & 46.5 & Intact & SB2 & Integrase, phage lysin, attachment invasion locus protein precursor \\
\hline P7 & 8 & Questionable & SB2 & Mobile element proteins \\
\hline P8 & 57 & Intact & SB2 and SB3 & Attachment invasion locus protein precursor, 2 tRNAs, phage lysis, acfC, and integrase \\
\hline P9 & 94.8 & Intact & SB4 and SB5 & Attachment invasion locus protein precursor, phage lysis 5 tRNAs, and integrase \\
\hline P10 & 24.8 & Incomplete & SB5 & Integrase and 3 tRNAs \\
\hline P11 & 54 & Intact & SB5 & Integrase, phage lysin, inv and 5 tRNAs \\
\hline P12 & 61.2 & Intact & SB5 & Attachment invasion locus protein precursor, integrase, yeeV, yeeX, stx2, and 3 tRNAs \\
\hline P13 & 24 & Intact & SB5 & Phage assembly proteins and Phage lysin \\
\hline P14 & 71.7 & Intact & SB5 & Integrase, attachment invasion locus protein precursor, and stx2 \\
\hline P15 & 77.2 & Questionable & SB5, SB6, and SB7 & Integrase, attachment invasion locus protein precursor, Phage lysin, and 4 tRNAs \\
\hline P16 & 23.9 & Questionable & SB7 & Phage lysin and integrase \\
\hline P17 & 13.1 & Questionable & SB7 & Mobile element proteins \\
\hline P18 & 20.5 & Questionable & SB7 & Integrase, yeeU, yeeV, espA, espB, espD, espF, sseE, yscL, escD, eae, and tir chaperone \\
\hline P19 & 21.7 & Questionable & SB7 & Integrase and phage eae \\
\hline
\end{tabular}

(71,736 bp), SS17 (71,730 bp), and, EC4115 (71,736 bp) were highly similar, while it was shorter in EDL933 (63,575 bp) (Figure 4). Next, the nucleotide sequences of the entire prophage genomes were compared by BLASTn. The similarity of the Stx2aencoding prophages was as follows; $99.98 \%$ for JEONG-1266 vs. SS17 (100\% coverage, 17 nucleotides difference, and 6 gaps), $100 \%$ for JEONG-1266 vs. EC4115 (100\% coverage, 1 nucleotide difference, and 0 gaps), and $97.60 \%$ for JEONG-1266 vs. EDL933 (74\% coverage) (Table 54 ). Stx2a-encoding prophage in JEONG1266, EC4115, and SS17 have 102 CDSs, while the one in EDL933 has 83 CDSs. Therefore, in silico PCR typing and prophage genome analysis together showed that JEONG-1266, SS17, and EC4115 are highly likely lysogenized by the same $\varphi s t x 2 a-\gamma$, indicating their strong potential to produce high levels of Stx2 toxins. Our data showed that the Stx2a-encoding prophage of EDL933 is structurally different from that of JEONG1266, SS17, and EC4115, although it was determined to be $\varphi$ stx $2 a-\gamma$ subtype.

\section{Shiga Toxin Production}

Stx production of JEONG-1266 was analyzed by MMC-mediated induction of the Stx-encoding prophages. The EDL933 strain was used as a positive control and $\mathrm{DH} 5 \alpha$, a stx gene-negative strain, was used as a negative control. The $\mathrm{OD}_{600}$ value of both JEONG-1266 and EDL933 decreased after MMC treatment, while the growth of $\mathrm{DH} 5 \alpha$ was not affected by MMC treatment (Figure 5A). This result indicates that bacterial cells of JEONG1266 and EDL933 were lysed by phage induction.

To investigate the production of Stx 2 toxins by JEONG1266, we conducted Western blot using Stx2-specific monoclonal antibodies in the presence and absence of MMC. Both JEONG1266 and EDL933 produced Stx2 toxins with MMC dependency
(Figure 5B); whether the toxins were Stx2a and/or Stx2c was not determined in this experiment since the Stx2-specific monoclonal antibody does not have differential specificities for Stx $2 \mathrm{a}$ and Stx $2 c$.

\section{Adhesion of JEONG-1266}

Adherence of the STEC O157 to the epithelial cells of the human intestinal tract is the initial step for its colonization and pathogenesis. STEC O157 EDL933 strain has been extensively studied in its ability to adhere to and colonize the human intestinal tract (Lewis et al., 2015; Cordonnier et al., 2017). To evaluate the adherence capability of JEONG-1266 to human cells, adherence assay was conducted using human HEp-2 cells with EDL933 and $\mathrm{DH} 5 \alpha$ as a positive and negative control, respectively. JEONG-1266 showed a significant increase in adherence to the HEp-2 cells $(P<0.05)$ compared with DH5 $\alpha$ (Figure 6). The adherence capability of JEONG-1266 was similar with that of EDL933 $(P>0.05)$, suggesting that JEONG-1266 is comparable to EDL933 in its ability to colonize the human intestinal tract.

\section{DISCUSSION}

To investigate the potential impact of an E. coli O157:H7 isolate which was a super-shedder origin on humans, we performed comparative genomics, phylogenetic analysis, and functional analyses and characterize the virulence potential. Phylogenetic analysis with $26 \mathrm{E}$. coli O157 strains from various sources showed that JEONG-1266 belongs to the prominent cluster including all spinach outbreak strains and super-shedder strains (Clade III, Figure 2A). In particular, JEONG-1266 formed a subclade with EC4115, a clinical isolate from the 2006 spinach 

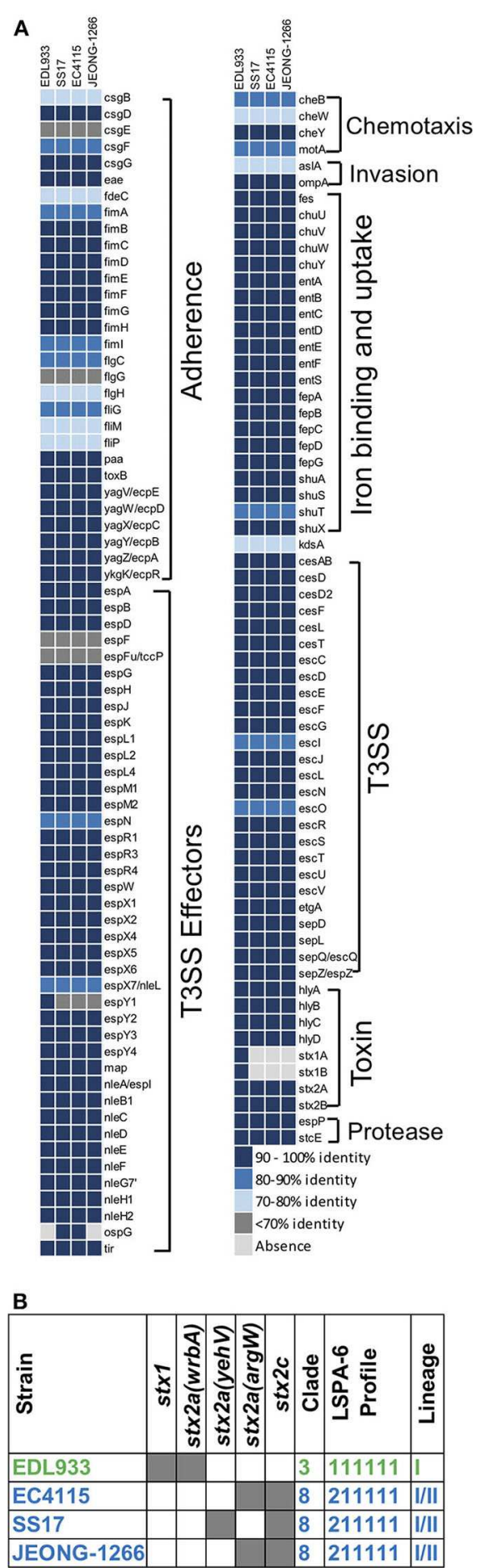

FIGURE 3 | Virulence gene profiles and genotyping of $E$. coli O157. (A) Virulence gene profile of JEONG-1266 and other E. coli O157 strains, SS17, EC4115, and EDL933. The virulence genes of 4 strains were identified by aligning their CDSs against the protein sequences in VFDB by BLASTp. Compared with the sequences of virulence genes in VFDB, virulence genes in 4 strains showed distinct identities that were displayed using 5 color codes. (B) Genotyping of stx subtypes, clade, and lineage of JEONG-1266 and other STEC 0157:H7 strains. A shaded gray box indicates the presence of the stx genes, and the insertion loci of the Stx2-encoding prophage. outbreak, and SS17, one of two super-shedder strains analyzed. The subsequent comparative analysis by Mauve revealed the significant collinearity in the whole-genome architecture between JEONG-1266, EC4115, and SS17 (Figure 2B). The genome of JEONG-1266 was shown to contain a total of 19 prophage regions. Despite prophage regions of E. coli O157:H7 genomes are typically hypervariable, those 19 prophage regions were shown to be highly conserved among the three strains, implying their close evolutionary relationships. This result strongly indicates the presence of an ecological connection between $E$. coli O157:H7 strains from super-shedders and clinical strains from the outbreak, which can represent a potential control point to prevent preharvest-stage transmission of E. coli O157.

Manning et al. identified the presence of hypervirulent subgroup, called Clade 8, of E. coli O157 associated with severe clinical symptoms such as HUS (Manning et al., 2008). Our data showed that JEONG-1266, together with EC4115 and SS17, belongs to Clade 8, highlighting the importance of the Clade 8 of bovine-origin. Clade 8 strains typically produce high levels of Stx toxins and are lysogenized with particular Stxencoding prophage subtypes (Ogura et al., 2015). To further understand the Stx-related genetic characteristics of JEONG1266, stx genotype and Stx2a-encoding prophage are analyzed. The finding that JEONG-1266, EC4115, and SS17 are lysogenized with the same subtype of Stx2a-encoding prophage, $\varphi s t x 2 a-\gamma$, implies that these strains have similar Stx-medicated cytotoxicity in humans despite their diverse origin (i.e., super-shedder cattle and clinical isolation). As the expression of the stx 2 genes is directly controlled by the lambdoid late promoter of the Stx2-encoding prophage (Waldor and Friedman, 2005), it is plausible that the subtype of an Stx2-encoding prophage is a critical determinant for Stx 2 production levels. It is worth note, however, that Stx2-encoding prophage subtypes $(\alpha, \beta, \gamma$, and $\delta)$ are classified by their well-conserved genome structure, their gene content can be variable within each subtype (Ogura et al., 2015), indicating in-depth genetic resolution of Stx2-encoding prophage may help better understanding of Stx production and virulence potential of E. coli O157:H7.

The whole genomes analysis revealed that genome-wide architecture (Figure 2B) and virulence gene profile (Figure 3A) are highly conserved among JEONG-1266, EC4115, and SS17 as well. This result is consistent with the previous study showing the similarity between SS17 and EC4115 genomes (Cote et al., 2015). There were only 48 SNPs in the core genome of JEONG-1266 in comparison to EC4115 (Figure S1). Both our phylogenetic data and the previous studies (Cote et al., 2015; Katani et al., 2017) clearly showed that SS17 and SS52 from super-shedders are phylogenetically closed to EC4115. As expected, JEONG-1266, SS17, and EC4115 strains shared a highly conserved virulence profile overall (Figure 3A); the most divergent gene categories $(<90 \%$ to the VFDB) include adherence and chemotaxis, both of which are typically related to the host specificity and environmental interactions. Consistently, our phenotypic analysis showed that JEONG-1266 displayed high capability in adherence to HEp-2 cells. Similar results were also observed by Cote et al. (2015) and Katani et al. (2015) when they evaluated the adherence capability of STEC O157:H7 from 


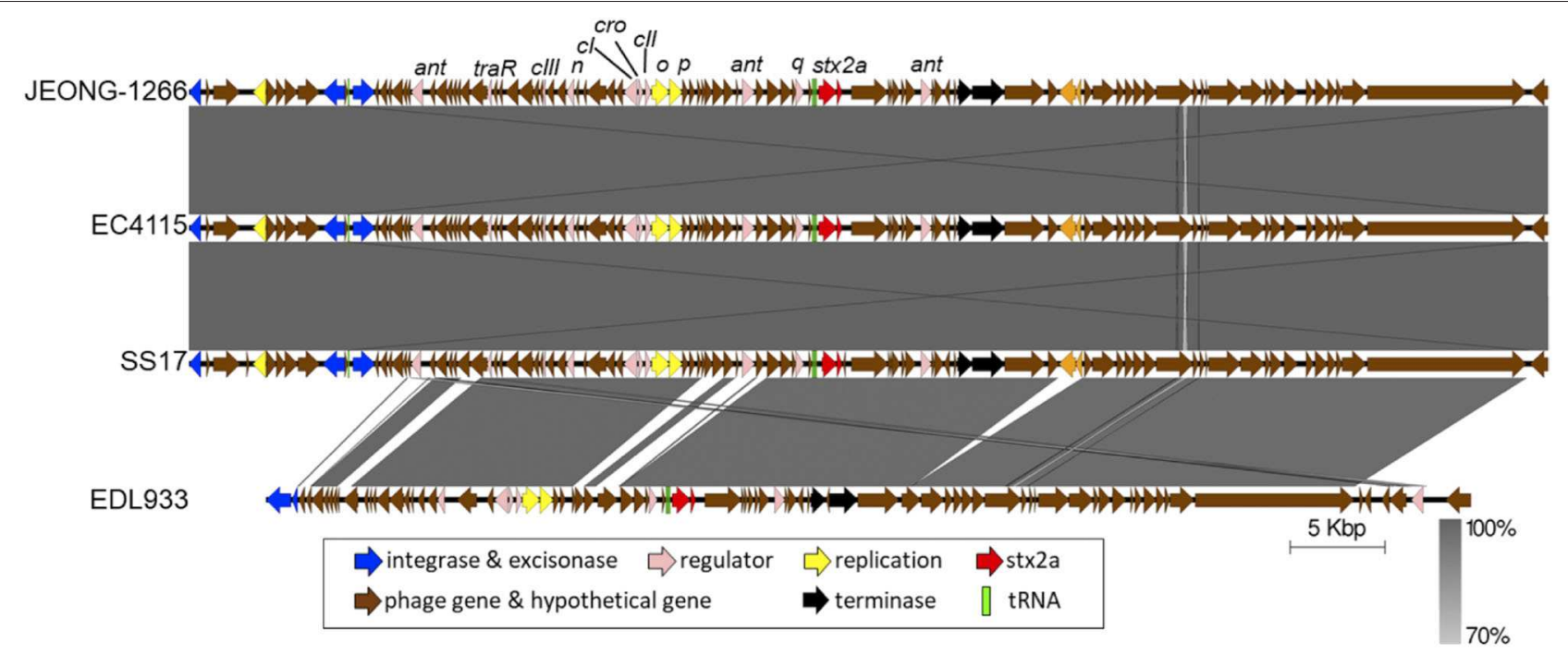

FIGURE 4 | Comparison of Stx2a-encoding prophages. The Stx2a-encoding prophage of JEONG-1266 was compared with the ones in EC4115, SS17, and EDL933. The CDS and tRNA were shown in colored arrows and blocks, respectively. The darkness of gray shade between strains reflects the similarity of the nucleotide sequences of Stx2a-encoding prophages.
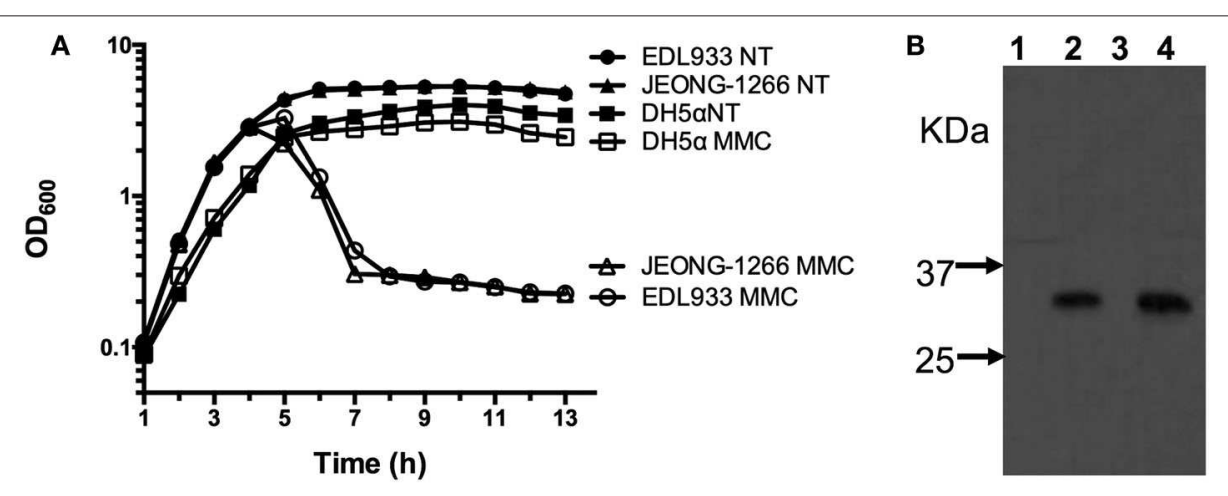

FIGURE 5 | Analyses of Shiga toxin production. (A) Survival curve of JEONG-1266, as well as EDL933 and DH5 $\alpha$, with (MMC) and without (NT) mitomycin C treatment. Mitomycin $\mathrm{C}$ was added to bacterial cell cultures when the $\mathrm{OD}_{600}$ reached 0.7. (B) Western blotting to detect the expression of Shiga toxin 2 in JEONG-1266 with and without mitomycin C treatment. EDL933 was used as a positive control. Samples from Lane 1 to 4 were EDL933 without MMC (lane 1), EDL933 with MMC (lane 2), JEONG-1266 without MMC (lane 3), and JEONG-1266 with MMC (lane 4).

super-shedders using bovine RAJ stratified squamous epithelial (RSE) cells. Those STEC O157:H7 from super-shedder cattle, including SS17 and SS52, formed aggregates surrounding the RSE cells. Given their genomic and genetic similarities, JEONG1266 may also have a similar super-shedder phenotype. Most importantly, the virulence profile result indicates that JEONG1266 possesses a comprehensive array of virulence genes required for the full virulence potential of STEC O157:H7, a characteristic that often lacks in strains of bovine origin.

Besides its cytotoxicity, Stx 2 has been implicated in enhancing adherence to and colonization of intestinal epithelial cells by E. coli O157:H7, possibly by increasing the expression of the host cell receptor for the pathogen (Robinson et al., 2006; Liu et al., 2010). Furthermore, the presence of stx2a was epidemiologically associated with the super-shedder of the pathogen by cattle (Matthews et al., 2013). Most recently, Fitzgerald el al. demonstrated that Stx2a has a critical role in animal-to-animal transmission of STEC and the development of super-shedders (Fitzgerald et al., 2019). Our data showed that JEONG-1226, together with EC4115 and SS17, carries stx2a and the Stx $2 \mathrm{a}$-encoding prophage subtype, $\varphi$ st $x 2 a-\gamma$, suggesting that the occurrence of such STEC subgroup in human clinical isolates and outbreaks may arise as a result of high cattle shedding levels associated with the $s t x$ genetic properties. Together, a dramatic combination of the super-shedder phenotype and hypervirulence potential of JEONG-1266 revealed by this study highlights the epidemiological and clinical importance of the STEC O157:H7 group of bovine origin. 


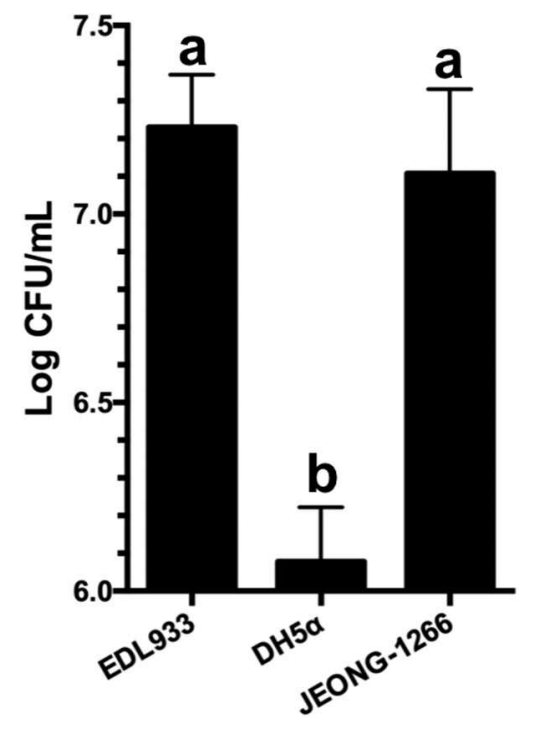

FIGURE 6 | Adherence of JEONG-1266 to HEp-2 cells. The adherence capability of JEONG-1266 to HEp-2 cells was compared with EDL933 (positive control) and $\mathrm{DH} 5 \alpha$ (negative control). The assay was performed in triplicate. Statistical analysis was conducted using a student's $t$-test $(\alpha=0.05)$. Means with different letters differ significantly.

\section{CONCLUSION}

In summary, the genetic and functional analyses revealed that E. coli O157:H7 strain JEONG-1266, isolated from supershedder cattle belongs to hypervirulent Clade 8, produces Stx2, shows high adherence capability, and contains major virulence genes typically present in clinical isolates. The hypervirulence potential of JEONG-1266 was also supported by the genome-wide phylogenetic and SNP analysis as well as highresolution subtyping of Stx-encoding prophage of the strain. An emerging subgroup of STEC O157:H7 with a combination of hypervirulence and super-shedder imposes a new threat for the public health and food industry. A better understanding of

\section{REFERENCES}

Alikhan, N. F., Petty, N. K., Ben Zakour, N. L., and Beatson, S. A. (2011). BLAST Ring Image Generator (BRIG): simple prokaryote genome comparisons. BMC Genom. 12:402. doi: 10.1186/1471-216412-402

Arthur, T. M., Ahmed, R., Chase-Topping, M., Kalchayanand, N., Schmidt, J. W., and Bono, J. L. (2013). Characterization of Escherichia coli O157: H7 strains isolated from supershedding cattle. Appl. Environ. Microbiol. 79, 4294-4303. doi: 10.1128/AEM.00846-13

Boerlin, P., Mcewen, S. A., Boerlin-Petzold, F., Wilson, J. B., Johnson, R. P., and Gyles, C. L. (1999). Associations between virulence factors of Shiga toxinproducing Escherichia coli and disease in humans. J. Clin. Microbiol. 37, 497-503. doi: 10.1128/JCM.37.3.497-503.1999

Brettin, T., Davis, J. J., Disz, T., Edwards, R. A., Gerdes, S., Olsen, G. J., et al. (2015). RASTtk: a modular and extensible implementation of the RAST algorithm for building custom annotation pipelines and annotating batches of genomes. Sci. Rep. 5:8365. doi: 10.1038/srep08365 bacterial genetics and host ecology of the specific subgroup of STEC may help us control the transmission of the pathogen at the pre-harvest level.

\section{DATA AVAILABILITY STATEMENT}

The datasets generated for this study can be found in the NCBI/NZ_CP014314/, https://www.ncbi.nlm.nih.gov/nuccore/ NZ_CP014314.1.

\section{ETHICS STATEMENT}

The animal study was reviewed and approved by University of Florida Institutional Animal Care and Use Committee (IACUC number 201003744).

\section{AUTHOR CONTRIBUTIONS}

LT, SL, DP, and KJ designed the study and drafted the manuscript. LT and SL performed the analyses. LT, DP, and KJ finalized the manuscript. KJ acquired funding.

\section{FUNDING}

This work was supported by the National Institute of Food and Agriculture, U.S. Department of Agriculture, under award number 2015-68003-22971 to KJ.

\section{ACKNOWLEDGMENTS}

We would like to thank Drs. Zhengxin Ma and Soojin Jeon for helping to edit the manuscript.

\section{SUPPLEMENTARY MATERIAL}

The Supplementary Material for this article can be found online at: https://www.frontiersin.org/articles/10.3389/fcimb. 2020.00271/full\#supplementary-material

Caprioli, A., Morabito, S., Brugere, H., and Oswald, E. (2005). Enterohaemorrhagic Escherichia coli: emerging issues on virulence and modes of transmission. Vet. Res. 36, 289-311. doi: 10.1051/vetres:2005002

CDC (2006). Ongoing multistate outbreak of Escherichia coli serotype O157:H7 infections associated with consumption of fresh spinach-United States, September 2006. Morb. Mortal. Wkly. Rep. 55, 1045-1046. Available online at: https://www.cdc.gov/mmwr/preview/mmwrhtml/mm5538a4.htm

Chase-Topping, M. E., Mckendrick, I. J., Pearce, M. C., Macdonald, P., Matthews, L., Halliday, J., et al. (2007). Risk factors for the presence of high-level shedders of Escherichia coli $\mathrm{O} 157$ on Scottish farms. J. Clin. Microbiol. 45, 1594-1603. doi: 10.1128/JCM.01690-06

Chen, L., Zheng, D., Liu, B., Yang, J., and Jin, Q. (2016). VFDB 2016: hierarchical and refined dataset for big data analysis-10 years on. Nucleic Acids Res. 44, D694-D697. doi: 10.1093/nar/gkv1239

Cordonnier, C., Etienne-Mesmin, L., Thevenot, J., Rougeron, A., Renier, S., Chassaing, B., et al. (2017). Enterohemorrhagic Escherichia coli pathogenesis: role of Long polar fimbriae in Peyer's patches interactions. Sci. Rep. 7:44655. doi: $10.1038 /$ srep 44655 
Cornick, N. A., Booher, S. L., and Moon, H. W. (2002). Intimin facilitates colonization by Escherichia coli $\mathrm{O} 157: \mathrm{H} 7$ in adult ruminants. Infect. Immun. 70, 2704-2707. doi: 10.1128/IAI.70.5.2704-2707.2002

Cote, R., Katani, R., Moreau, M. R., Kudva, I. T., Arthur, T. M., DebRoy, C., et al. (2015). Comparative analysis of super-shedder strains of Escherichia coli O157: $\mathrm{H} 7$ reveals distinctive genomic features and a strongly aggregative adherent cells. PloS one. 10:e0116743.doi: 10.1371/journal.pone.0116743

Croxen, M. A., Law, R. J., Scholz, R., Keeney, K. M., Wlodarska, M., and Finlay, B. B. (2013). Recent advances in understanding enteric pathogenic Escherichia coli. Clin. Microbiol. Rev. 26, 822-880. doi: 10.1128/CMR.00022-13

Darling, A. C., Mau, B., Blattner, F. R., and Perna, N. T. (2004). Mauve: multiple alignment of conserved genomic sequence with rearrangements. Genome Res. 14, 1394-1403. doi: 10.1101/gr.2289704

Darling, A. E., Mau, B., and Perna, N. T. (2010). ProgressiveMauve: multiple genome alignment with gene gain, loss and rearrangement. PLoS One 5:e11147. doi: 10.1371/journal.pone.0011147

Eppinger, M., Mammel, M. K., Leclerc, J. E., Ravel, J., and Cebula, T. A. (2011). Genomic anatomy of Escherichia coli O157:H7 outbreaks. Proc. Natl. Acad. Sci. U. S. A. 108, 20142-20147. doi: 10.1073/pnas.1107176108

Fitzgerald, S. F., Beckett, A. E., Palarea-Albaladejo, J., Mcateer, S., Shaaban, S., Morgan, J., et al. (2019). Shiga toxin sub-type 2a increases the efficiency of Escherichia coli $\mathrm{O} 157$ transmission between animals and restricts epithelial regeneration in bovine enteroids. PLoS Pathog. 15:e1008003. doi: 10.1371/journal.ppat.1008003

Garmendia, J., Ren, Z., Tennant, S., Midolli Viera, M. A., Chong, Y., Whale, A., et al. (2005). Distribution of tccP in clinical enterohemorrhagic and enteropathogenic Escherichia coli isolates. J. Clin. Microbiol. 43, 5715-5720. doi: 10.1128/JCM.43.11.5715-5720.2005

Griffin, P. M., and Tauxe, R. V. (1991). The epidemiology of infections caused by Escherichia coli $\mathrm{O} 157: \mathrm{H} 7$, other enterohemorrhagic E. coli, and the associated hemolytic uremic syndrome. Epidemiol. Rev. 13, 60-98. doi: 10.1093/oxfordjournals.epirev.a036079

Grys, T. E., Siegel, M. B., Lathem, W. W., and Welch, R. A. (2005). The StcE protease contributes to intimate adherence of enterohemorrhagic Escherichia coli $\mathrm{O} 157: \mathrm{H7}$ to host cells. Infect. Immun. 73, 1295-1303. doi: 10.1128/IAI.73.3.1295-1303.2005

Gyles, C. L. (2007). Shiga toxin-producing Escherichia coli: an overview. J. Anim. Sci. 85, E45-E62. doi: 10.2527/jas.2006-508

Heiman, K. E., Mody, R. K., Johnson, S. D., Griffin, P. M., and Gould, L. H. (2015). Escherichia coli $\mathrm{O} 157$ outbreaks in the United States, 2003-2012. Emerg. Infect. Dis. 21, 1293-1301. doi: 10.3201/eid2108.141364

Hoffmann, S., Batz, M. B., and Morris, J. G. Jr. (2012). Annual cost of illness and quality-adjusted life year losses in the United States due to 14 foodborne pathogens. J. Food Prot. 75, 1292-1302. doi: 10.4315/0362-028X.JFP-11-417

Iyoda, S., Manning, S. D., Seto, K., Kimata, K., Isobe, J., Etoh, Y., et al. (2014). Phylogenetic clades 6 and 8 of enterohemorrhagic Escherichia coli O157:H7 with particular stx subtypes are more frequently found in isolates from hemolytic uremic syndrome patients than from asymptomatic carriers. Open Forum Infect. Dis. 1:ofu061. doi: 10.1093/ofid/ofu061

Jaureguy, F., Landraud, L., Passet, V., Diancourt, L., Frapy, E., Guigon, G., et al. (2008). Phylogenetic and genomic diversity of human bacteremic Escherichia coli strains. BMC Genom. 9:560. doi: 10.1186/1471-2164-9-560

Jeon, S. J., Elzo, M., Dilorenzo, N., Lamb, G. C., and Jeong, K. C. (2013). Evaluation of animal genetic and physiological factors that affect the prevalence of Escherichia coli O157 in cattle. PLoS One. 8:e55728. doi: 10.1371/journal.pone. 0055728

Jeon, S. J., Oh, M., Yeo, W. S., Galvao, K. N., and Jeong, K. C. (2014). Underlying mechanism of antimicrobial activity of chitosan microparticles and implications for the treatment of infectious diseases. PLoS One 9:e92723. doi: 10.1371/journal.pone.0092723

Katani, R., Cote, R., Kudva, I. T., Debroy, C., Arthur, T. M., and Kapur, V. (2017). Comparative genomics of two super-shedder isolates of Escherichia coli O157: H7. PLoS One 12:e0182940. doi: 10.1371/journal.pone.0182940

Katani, R., Cote, R., Raygoza Garay, J. A., Li, L., Arthur, T. M., Debroy, C., et al. (2015). Complete genome sequence of SS52, a strain of Escherichia coli O157:H7 recovered from supershedder cattle. Genome Announc. 3:e01569-14. doi: 10.1128/genomeA.01569-14
Kulasekara, B. R., Jacobs, M., Zhou, Y., Wu, Z., Sims, E., Saenphimmachak, C., et al. (2009). Analysis of the genome of the Escherichia coli O157:H7 2006 spinach-associated outbreak isolate indicates candidate genes that may enhance virulence. Infect. Immun. 77, 3713-3721. doi: 10.1128/IAI.00198-09

Laing, C. R., Buchanan, C., Taboada, E. N., Zhang, Y., Karmali, M. A., Thomas, J. E., et al. (2009). In silico genomic analyses reveal three distinct lineages of Escherichia coli O157:H7, one of which is associated with hyper-virulence. BMC Genom. 10:287. doi: 10.1186/1471-2164-10-287

Lewis, S. B., Cook, V., Tighe, R., and Schuller, S. (2015). Enterohemorrhagic Escherichia coli colonization of human colonic epithelium in vitro and ex vivo. Infect. Immun. 83, 942-949. doi: 10.1128/IAI.02928-14

Liu, B., Yin, X., Feng, Y., Chambers, J. R., Guo, A., Gong, J., et al. (2010). Verotoxin 2 enhances adherence of enterohemorrhagic Escherichia coli O157:H7 to intestinal epithelial cells and expression of beta 1-integrin by IPEC-J2 cells. Appl. Environ. Microbiol. 76, 4461-4468. doi: 10.1128/AEM.00182-10

Liu, K., Knabel, S. J., and Dudley, E. G. (2009). rhs genes are potential markers for multilocus sequence typing of Escherichia coli O157:H7 strains. Appl. Environ. Microbiol. 75, 5853-5862. doi: 10.1128/AEM.00859-09

Manning, S. D., Motiwala, A. S., Springman, A. C., Qi, W., Lacher, D. W., Ouellette, L. M., et al. (2008). Variation in virulence among clades of Escherichia coli O157:H7 associated with disease outbreaks. Proc. Natl. Acad. Sci. U. S. A 105, 4868-4873. doi: 10.1073/pnas.0710834105

Matthews, L., Low, J. C., Gally, D. L., Pearce, M. C., Mellor, D. J., Heesterbeek, J. A., et al. (2006). Heterogeneous shedding of Escherichia coli O157 in cattle and its implications for control. Proc. Natl. Acad. Sci. U. S. A 103, 547-552. doi: $10.1073 /$ pnas. 0503776103

Matthews, L., Reeve, R., Gally, D. L., Low, J. C., Woolhouse, M. E., Mcateer, S. P., et al. (2013). Predicting the public health benefit of vaccinating cattle against Escherichia coli O157. Proc. Natl. Acad. Sci. U. S. A 110, 16265-16270. doi: $10.1073 /$ pnas.1304978110

Mckee, M. L., Melton-Celsa, A. R., Moxley, R. A., Francis, D. H., and O'brien, A. D. (1995). Enterohemorrhagic Escherichia coli O157:H7 requires intimin to colonize the gnotobiotic pig intestine and to adhere to HEp-2 cells. Infect. Immun. 63, 3739-3744. doi: 10.1128/IAI.63.9.3739-3744.1995

Mead, P. S., Slutsker, L., Dietz, V., Mccaig, L. F., Bresee, J. S., Shapiro, C., et al. (1999). Food-related illness and death in the United States. Emerg. Infect. Dis. 5, 607-625. doi: 10.3201/eid0505.990502

Melton-Celsa, A., Mohawk, K., Teel, L., and O'brien, A. (2012). Pathogenesis of Shiga-toxin producing Escherichia coli. Curr. Top. Microbiol. Immunol. 357, 67-103. doi: 10.1007/82_2011_176

Melton-Celsa, A. R. (2014). Shiga Toxin (Stx) classification, structure, and function. Microbiol. Spectr. 2:EHEC-0024-2013. doi: 10.1128/microbiolspec.EHEC-0024-2013

Munns, K. D., Selinger, L. B., Stanford, K., Guan, L., Callaway, T. R., and Mcallister, T. A. (2015). Perspectives on super-shedding of Escherichia coli O157:H7 by cattle. Foodborne Pathog. Dis.12, 89-103. doi: 10.1089/fpd.2014.1829

Nataro, J. P., and Kaper, J. B. (1998). Diarrheagenic Escherichia coli. Clin. Microbiol. Rev. 11, 142-201. doi: 10.1128/CMR.11.1.142

Neupane, M., Abu-Ali, G. S., Mitra, A., Lacher, D. W., Manning, S. D., and Riordan, J. T. (2011). Shiga toxin 2 overexpression in Escherichia coli O157:H7 strains associated with severe human disease. Microb. Pathog. 51, 466-470. doi: 10.1016/j.micpath.2011.07.009

Ogura, Y., Mondal, S. I., Islam, M. R., Mako, T., Arisawa, K., Katsura, K., et al. (2015). The Shiga toxin 2 production level in enterohemorrhagic Escherichia coli O157:H7 is correlated with the subtypes of toxin-encoding phage. Sci. Rep. 5:16663. doi: $10.1038 /$ srep 16663

Overbeek, R., Olson, R., Pusch, G. D., Olsen, G. J., Davis, J. J., Disz, T., et al. (2014). The SEED and the Rapid annotation of microbial genomes using subsystems technology (RAST). Nucleic Acids Res. 42, D206-D214. doi: 10.1093/nar/gkt1226

Page, A. J., Cummins, C. A., Hunt, M., Wong, V. K., Reuter, S., Holden, M. T., et al. (2015). Roary: rapid large-scale prokaryote pan genome analysis. Bioinformatics 31, 3691-3693. doi: 10.1093/bioinformatics/btv421

Park, D., Stanton, E., Ciezki, K., Parrell, D., Bozile, M., Pike, D., et al. (2013). Evolution of the Stx2-encoding prophage in persistent bovine Escherichia coli O157:H7 strains. Appl. Environ. Microbiol. 79, 1563-1572. doi: 10.1128/AEM.03158-12 
Paton, J. C., and Paton, A. W. (1998). Pathogenesis and diagnosis of Shiga toxin-producing Escherichia coli infections. Clin. Microbiol. Rev. 11, 450-479. doi: 10.1128/CMR.11.3.450

Pollock, K. G., Beattie, T. J., Reynolds, B., Stewart, A., and Cowden, J. M. (2007). Clinical management of children with suspected or confirmed E. coli O157 infection. Scott. Med. J. 52, 5-7. doi: 10.1258/rsmsmj.52.3.5

Robinson, C. M., Sinclair, J. F., Smith, M. J., and O’brien, A. D. (2006). Shiga toxin of enterohemorrhagic Escherichia coli type O157:H7 promotes intestinal colonization. Proc. Natl. Acad. Sci. U. S. A 103, 9667-9672. doi: $10.1073 /$ pnas.0602359103

Sakuma, M., Urashima, M., and Okabe, N. (2006). Verocytotoxin-producing Escherichia coli, Japan, 1999-2004. Emerg. Infect. Dis. 12, 323-325. doi: 10.3201/eid1202.050268

Schmitt, C. K., Mckee, M. L., and O’brien, A. D. (1991). Two copies of Shiga-like toxin II-related genes common in enterohemorrhagic Escherichia coli strains are responsible for the antigenic heterogeneity of the O157:H- strain E32511. Infect. Immun. 59, 1065-1073. doi: 10.1128/IAI.59.3.1065-1073.1991

Seemann, T. (2014). Prokka: rapid prokaryotic genome annotation. Bioinformatics, 30, 2068-2069. doi: 10.1093/bioinformatics/btu153

Sheng, H. Q., Lim, J. Y., Knecht, H. J., Li, J., and Hovde, C. J. (2006). Role of Escherichia coli O157: H7 virulence factors in colonization at the bovine terminal rectal mucosa. Infect. Immun. 74, 4685-4693. doi: 10.1128/IAI.00406-06

Shridhar, P. B., Patel, I. R., Gangiredla, J., Noll, L. W., Shi, X. R., Bai, J. F., et al. (2018). Genetic analysis of virulence potential of Escherichia coli O104 serotypes isolated from cattle feces using whole genome sequencing. Front. Microbiol. 9:341. doi: 10.3389/fmicb.2018.00341

Stanton, E., Park, D., Dopfer, D., Ivanek, R., and Kaspar, C. W. (2014). Phylogenetic characterization of Escherichia coli O157: H7 based on IS629 distribution and Shiga toxin genotype. Microbiology 160, 502-513. doi: 10.1099/mic.0.073437-0

Stothard, P., and Wishart, D. S. (2005). Circular genome visualization and exploration using CGView. Bioinformatics 21, 537-539. doi: 10.1093/bioinformatics/bti054

Sullivan, M. J., Petty, N. K., and Beatson, S. A. (2011). Easyfig: a genome comparison visualizer. Bioinformatics 27, 1009-1010. doi: 10.1093/bioinformatics/btr039

Teng, L., Ginn, A., Jeon, S., Kang, M., and Jeong, K. C. (2016). Complete genome sequence of an Escherichia coli O157:H7 strain isolated from a super-shedder steer. Genome Announc. 4:e00258-16. doi: 10.1128/genomeA.00258-16

Treangen, T. J., Ondov, B. D., Koren, S., and Phillippy, A. M. (2014). The Harvest suite for rapid core-genome alignment and visualization of thousands of intraspecific microbial genomes. Genome Biol. 15:524. doi: 10.1186/s13059-014-0524-x

Tyler, J. S., Mills, M. J., and Friedman, D. I. (2004). The operator and early promoter region of the Shiga toxin type 2-encoding bacteriophage 933W and control of toxin expression. J. Bacteriol. 186, 7670-7679. doi: 10.1128/JB.186.22.7670-7679.2004

Waldor, M. K., and Friedman, D. I. (2005). Phage regulatory circuits and virulence gene expression. Curr. Opin. Microbiol. 8, 459-465. doi: 10.1016/j.mib.2005.06.001

Wattam, A. R., Abraham, D., Dalay, O., Disz, T. L., Driscoll, T., Gabbard, J. L., et al. (2014). PATRIC, the bacterial bioinformatics database and analysis resource. Nucleic Acids Res. 42, D581-D591. doi: 10.1093/nar/ gkt1099

Wells, J. G., Shipman, L. D., and Greene, K. D. (1991). Isolation of Escherichia coli serotype O157: $\mathrm{H} 7$ and other Shiga-like-toxin-producing E. coli from dairy cattle. J. Clin. Microbiol. 29, 985-989. doi: 10.1128/JCM.29.5.985-98 9.1991

Wirth, T., Falush, D., Lan, R. T., Colles, F., Mensa, P., Wieler, L. H., et al. (2006). Sex and virulence in Escherichia coli: an evolutionary perspective. Mol. Microbiol. 60, 1136-1151. doi: 10.1111/j.1365-2958.2006.0 5172.x

Yang, Z., Kovar, J., Kim, J., Nietfeldt, J., Smith, D. R., Moxley, R. A., et al. (2004). Identification of common subpopulations of non-sorbitol-fermenting, beta-glucuronidase-negative Escherichia coli O157:H7 from bovine production environments and human clinical samples. Appl. Environ. Microbiol. 70, 6846-6854. doi: 10.1128/AEM.70.11.6846-6854.2004

Zhou, Y., Liang, Y., Lynch, K. H., Dennis, J. J., and Wishart, D. S. (2011). PHAST: a fast phage search tool. Nucleic Acids Res. 39, W347-W352. doi: $10.1093 /$ nar/gkr485

Conflict of Interest: The authors declare that the research was conducted in the absence of any commercial or financial relationships that could be construed as a potential conflict of interest.

Copyright (c) 2020 Teng, Lee, Park and Jeong. This is an open-access article distributed under the terms of the Creative Commons Attribution License (CC BY). The use, distribution or reproduction in other forums is permitted, provided the original author(s) and the copyright owner(s) are credited and that the original publication in this journal is cited, in accordance with accepted academic practice. No use, distribution or reproduction is permitted which does not comply with these terms. 